Monatsschrift f. Geburtshülfe u. Gynäkologie 1926;72:247-252

\title{
IV. Buchbesprechungen
}

Hugo Sellheim, Die geburtshílflich-gijnäkologische Untersuchung. Bin Leit-faden für Studierencle und praktische Ärzte. 4. Aufl. München 1923. J. F. Bergmann.

Wem die Aufgabe zufällt, Studierenden und heranwachsenden Ärzten zunächst einmal clas gute und sachgemäße Untersuchen zu lehren, der wird die neue Aiiñage des Sellheimschen Leitfadens freudigst begrüßen. Das Buch erscheint in seinen bekannten Vorzügen zur rechten Zeit auf clem Plane, die übereilte und gewiß nicht immer grünclliche Ausbildung cler jungen Ärzteschaft in der Xachkriegszeit hat gar keine Muße gehabt, um geracle das notwencligste Rüstzeug, , die Untersuchung", in der un-bedingt erforderlichen Gründlichkeit zu berücksichtigen. Das erfährt man leider recht häufig. Da muß es um so dankbarer begrüßt werden, wenn unser Meister cler Dai-stellungskunst mit einer neuen Auflage auf denI Plane erschienen ist.

Als Meister hat sich S. schon allein dadurch bewährt, daß er eine Reihe von früheren Abbildangen fortgelassen hat, um durch ein , Zuviel" nicht zu verwirren. Wenige neue Zeichnungen sind hinzugekommen. Von cliesen sind besonders hervorzaheben: die schematische Darstellung der Haltimg des vorangehenden Fruchtpoles, die einfache Scheidenuntersuchung mit Respektierung cles Muttermundes und unter Eindringen in den Mutter-mund sowie die Dilatation mit Hegarstiften.

Neu aufgenommen sind in die 4. Auflage unter ancleren Abschnitten: Psychologie im Umgange mit kranken Frauen, Mastdarmuntersuchung, Scheidenuntersuchung, die anatomischen Grundlagen für clas Tasterkennen an den weiblichen Unterleibsorganen, Luftfüllung des Bauches von einer Punktionsöffnung oder vom Mattermunde her zii diagnostischen Zwecken und anderes.

Gerade in den neu geschaffenen Abschnitten wird auch cler Erfahrenere eine Fülle von Anregungen und Belehrungen finden, welche dankbarst zu begrüßen sind.

248

-Buchbesprechungen.

Auch diese Auflage wírd, wie die voraufgegangenen, schnell ihren Weg zu einem dankbaren Leserkreis finden.

Trotz der Ungunst der Zeiten hat der Verlag es nicht gescheut, in fast vorkriegszeitlicher Ausrüstung das Buch auf seine Lebensbahn zu senden.

Ed. Marfin-Elberfeld.

Sellheim, H., Das Geheimnis vom Ewig-Weiblichen. 2. umgearbeitete und vermehrte Aiiflage. Stuttgart 1924, Ferd. Enke.

In der 2. Auflage faßt Verfasser verschiedene Vorträge über Frauen-kunde zusammen, welche zerstreut seit dem Erscheinen der 1. Auflage er-schienen sind. Es sind ferner die Vorträge, welche im ,,Reize der Frau" und ,Geheimnis des Ewig-Weiblichen"” veröffentlicht waren, zusammen-genommen worden. S. hat schließlích in dieser 2. Auflage das niedergelegt, was er gelegentlich in kleinem Kreise vorgetragen hat. Auf diese Weise ist ein Buch entstanden, welches uns wieder von neuem den Vorkämpfer einer ,,Frauenkunde” in modernstemAusmaße vor Augen führt. Die ruhige, klare Auffassung berührt wohltuend im Vergleich zu den 
außerordentlich vielen Autoren, welche ähnliche Gedanken mit krampfhaftem Beiwerk glauben schmücken zu müssen. Das neue Werk Sellheims wird nicht ver-fehlen, nach den verschiedensten Richtungen hin belehrend zu wirken und so seinen gegebenen Weg zu nehnien. Der Verlag hat durch würdige Ausgestaltung das Seine getan, um den

Erfo $1 / 8$ zu sichern. Ed. Martin.

Franz, K., u. B. Zondek, Beziehungen der Geburtshilfe und Gynäkologie zur inneren Medizin. Spezielle Palhologie und Therapie inner er Krank-heiten. Krauß u. Brugsch. Berlin-Wien, Urban u. Schwarzenberg. Angesichts der weitreichenden Forschungen, welche in den letzten Jahrzehnten unsere Kenntnisse der Beziehungen unseres Fachs zur inneren Medizin klarzustellen und zu vertiefen bestrebt waren, ist diese Arbeit ein sehr lebhaft zu begrüßendes Unternehmen. Die Verfasser haben sie im Rahmen des Krauß-Brugschschen Handbuches durchgeführt. Gestützt auf das große Material der Frauenklinik der Charitá und der vielfältigen Vorstudien, über welche besonders Herr B. Zondek zum Teil schon berichtet hat, gebieten die Verf. über eine hohe Autorität, die sicher allseitig ihren Schlüssen zuerkannt wird. Sie bringen die Krankheiten des Herzens, des Blutes, der Nieren, des Stoffwechsels und der endokrinen Drüsen von diesem Gesichtspunkte aus zur Darstellung. Man muß ihnen beistimmen, wenn sie auf die Erörterung der Krankheiten der Leber zunächst verzichtet haben, weil keine besonderen Beziehungen zur Frauenheilkunde bestehen und das, was in Beziehung zur Schwangerschaft gebracht werden kann, zu gering und lückenhaft ist. Es entspricht der ernsten kritischen Auffassung der Verff., daß sie nur das, was wissenschaftlich einigermaßen feststeht, bringen, und was therapeutische Schlußfolgerungen zuläßt: daraus lernt nicht nur der praktische Arzt, auch der Interne und der Frauenarzt.

Unter dem Kapitel vom Herzen stellen die Verf. fest, daß keinerlei Tatsachen vorhanden sind, welche darauf hinweisen, daß Herz- und Ge-fäßsystem des Weibes spezifisch-funktionelle Eigenschaften haben, daß ein geringer anatomischer Unterschied zwischen dem Herzen des Mamies und der Frau besteht. Ref. muß darauf verzichten, auf alle die ihn lebhaft Buchbesprechungen.

249

interessierenden Einzelheiten einzugehen. Er will nur hervorheben, daß nach dem Ergebnis dieser Uhtersuchungen bisher keinerlei Beweis erbracht ist, daß das Herz durch ein Myom allein krank wird: eine Feststellung, welche die vielseitig bisher festgehaltene Auffassung umstößt. Das Hauptkontingent der an Mitralfehlern ziigrundegehenden ist der Mitralstenose allein oder ihrer Kombination mit Mitralinsuffizienz zur Last zu legen. In jedem Fall@ ist das Verhalten des Herzmuskels von ausschlaggebender Bedeutung. Gegenüber abweichenden Ansichten mahnen die Verff. bei herzkranken Schwangeren zur Aufmerksamkeit und Vorsicht.

In der Frage der Lungentuberkulose wird ausdrücklich betont, daß sie nicht zahlenmäßig zu lösen ist. Das bezieht sich wohl nach allgemeiner Übereinstimmung auf die manifesten Erkrankungen: im ersten Stadium wird die Schwangerschaftsunterbrechung empfohlen; im zweiten hat sie wenig Aussicht auf Erfolg; im dritten sind die Aussichten schlecht. Die Unterbrechung darf nur als prophylaktische Maßnahme angegeben werden; die Patienten sind danach dem Internisten zuzufiihren. Die Unterbrechung ist nur in den ersten vier Monaten zu empfehlen. Die Prognose des Kindes ist ungünstig: Stillverbot. Antikonzeptionelle Mittel. Vorsicht mit der Sterilisierung! An dieser Stelle bätte B, ef. gern ein Urteil über die temporäre Sterilisierung durch Bestrahlung gefunden, schon im Hinblick darauf, daß von ihnen die supravaginale Korpusamputation als das Verfahren der Wahl anempfohlen wird. 
Sehr eingehend werden die unzweifelhaft nachweisbaren Veränderungen im hämatopoetischen System der Frau während der Gestationsperiode erörtert. Dieselben erscheinen als der Ausdruck der biologischen Urn-stellung, die der Körper durch die Gravidität erfährt. Chlorose, Hämophilie, perniziöse Anämie werden besonders in ihrer Bedeutung für die Kompli-kation mit Schwangerschaft besprochen.

Die Annahme einer spezifischen Xierenerkrankung der Schwangerschaft wird abgelehnt: es handelt sich nur um Symptomenkomplexe: Albuminnrie, Hydrops gravidarum, Hypertonie, Sehstörungen. Die das Krankheitsbikl bedingende Noxe muß beim Stoffaustausch zwischen Mutter und Kind entstehen, alle Symptome gehen in kürzester Zeit restlos zurück, sobald der Stoffaustausch nicht mehr notwendig, toxische Sub-stanzen nicht mehr gebildet werden. Hydrops, Hypertonie und Albuminurie sind Begleiterscheinungen der Gravidität. Sie gehören zum Symptomen-komplex der Eklampsie. Es erscheint zu weitgehend, ein Symptom wie den Hydrops als Ursache der Eklampsie zu betrachten. Das Schwangerschafts-gift ist noch unbekannt; nach allem ist der Angriffspunkt das Ge $\Lambda$ vebe. Verf. prägen dafür die Bezeichnung Histopathia gravidarum. Die Schwanger-schaftstoxikose ist nur der Ausdruck der erhöhten Schwangerschafts-gewebeschädigung. Die verschiedenen Organe können in bunter Reihen-folge betroffen werden, so daß die klinischen Symptome in alien möglichen Variationen auftre

Der Begriff Schwangerschaftsnephrose muß fallen.

Nach eingehender Klarstellung des Stoffwechsels wird erörtert, wie weit Generationsvorgänge denselben in deutlicher Weise beeinflussen. Unter den Stoffwechselkrankheiten wird zunächst der Diabetes in Beziehung zur Menstruation, Libido, Fertilität und Schwangerschaft, dann zur Fett-sucht und zur Gicht besprochen.

250

Buchbesprecliungen.

Verf. berichten eingehend über die Lehre von der inneren Sekretion des Ovarium, über Veränderungen des endokrinen Systems in der Schwanger-schaft und bei Erkrankungen der weiblichen Sexualorgane. Auf die einzelnen Generationsphasen, die gerade durch Forschung der letzten Jahre näher geklärt sind, wird ausführlicher eingegangen. - Der gelbe Körper wircl als endokrine Druse aufgefaßt, die den genitalen Turgor der Generations jahre aufrechterhält und den vierwöchentlichen menstruellen Zyklus bewirkt. Mit Rob. Meyer und Labhardt betrachten Verf. die Menstruation als einen von der Natur beabsichtigten Vorgang. Alle Umwandlungen der Sehleim-haut des Uterus dienen nicht dazu, die Menstruation zu veranlassen, sondern eine Schwangerschaft zu ermöglichen. Eine selbständige inter -stitielle Druse wird beim Menschen negiert. Die Eizelle spielt die wichtigste Rolle als Regulator der gesamten Generationsvorgänge. Ovulation, Corpus luteum und Menstruation bilden eine biologische Einheit. Als Störungen der Ovarialfunktion und ihrer Wirkung auf die Sexualorgane werclen die ovariellen Blutungen, Dys $\pi$ ienorrhöe, Amenorrhöe, in der Auswirkung auf den Gesamtorganismus die Wellenbewegung im weiblichen Organismus, auf die Funktion entfernter Organe besprochen. Unter diesen begegnen wir als einem ISfovum einer ovariellen Dermatose. Die klimakterischen Wallungen werden als pathologische, auf einer sekretorischen Stoning beruhende Reize des Vasomot·orenzentrum aufgefaßt. Die Generations-drüse ruptiert auch die Knochenformen. Eunuchoidismus und Infantilismus, Hermaphroditismus und Pseudohermaphroditismus sind Auswirkungen der Störungen der Druse. Die Kenntnisse über die Beziehungen der anderen endokrinen Drüsen zu den Gestationsvorgängen sind nur gering. Die Organtherapie wird nur in der Form der Organtransplantation anerkannt; nur sie kann eine gewisse substituierende Wirkung haben. 
Dieser erzwungen kurze Bericht läßt die staunenswerte Fülle des

Gegebenen erkennen. Die mis alien oft überraschenden Deutungen so vieler Vorgänge wird so überzeugend vorgetragen und so einleuchtend belegt, daß Ref. nur sein lebhaftes Bedauern ausdrücken kann, daß diese höchst wertvolle Gabe nicht ohne das Gesamthandbuch allgemein zugänglich sein soil. Ref. kann es sich nicht versagen, den Herren Verfassern lebhaft für den hohen Genuß zu danken und die tiefwirkende Anregung den weiteren Forschungen auf diesem Gebiet mit gespanntester Aufmerksamkeit zu folgen. A. Martin.

Sserdjuko $\Lambda$ V, Zur Frage der funktíonellen Beziehungen zwischen dem Drüsen-paranchym des Ovariums und der Nebennierenrinde. Moskau 1924.

Nach S. besteht eine funktionelle Abhängigkeit zwischen der sekre torischen Funktion der îCebennierenrinde, des Parenchym, des Corpus luteum und der Druse des Ovariums. Sie dokumentiert sich in Ver änderungen der Zellen sekretorischen Charakters, welche nach seinen Experimenten eine vikariierende Rolle zwischen den drei Drüsen spielen. Für den Funktionssynergismus spricht der Charakter ihres Sekrets. Die Produktion des lipoiden Sekrets steigert sich in den N1/8bennieren bei einer sekretorischen Funktionsstörung der interstitiellen Druse des Corpus luteum und umgekehrt. A. Martin.

BuchbesprechuDgen.

251

I). Th. Malade, Semmelweis, Der Reíter der Mutter. Der Roman eines ärzt-liclien Lebens. München 1924. J. F. Lehmann.

Eine Huldigung für Semmelweis ist in jeder Gestalt berechtigt und um so mehr, wenn sie in so künstlerischer Form dargebracht wird. Der Lebensgang und die seelische Entwícklung S. sind in packender Weise geschildert. Dabei muß die Verallgemeinerung der Erkenntnis des Kindbettfiebers in Laienkreisen als selbstverständlich betont werden. Möge das Buch auch in diesen eine recht ausgedehnte Verbreitung finden.

A. Martin.

Hubert Peters, Kompendium der Geburtshílfe (Propcideutik und Operations-lehre). Wien 1925.

F. Deuticke.

Das Ziel des Verf., eine kurz gehaltene Propädentik und Operationslehre für den Studenten und den angehenden Arzt in der Praxis zu schaffen, erscheint in bester Form erreicht: die knappe Darstellung ist so klar, sagt in aller Kürze so viel, daß der Ratsuchende in der Tat schnell und sicher orientiert wird. Das Büchlein ist eine sehr empfehlungswerte Ergänzung zu unseren so hervorragenden eingehenden Lehrbüchern. Ref. wünscht ihm lebhaft einen besseren und großen Erfolg - gegenüber dem Mißerfolg, den sein eigenes ähnliches Büchlein (1877 bei Hirschwald erschienen) gehabt hat! A. Martin.

Kurt Wiener, Die Beziehungen der Genitalorgane zu Hautverunderungen. Halle 1924, Carl Marhold.

Verf. gibt einen Überblick über den gegenwärtigen Stand unserer

Kenntnis dieser Zusammenhänge an Hand eines sehr genauen Literaturstudium und eigener Fälle aus der Breslauer Hautklinik (Jadassohn). Im 
Kapitel ,, Menstruation und Haut'” werden die Fragen der inneren Sekretion, der Dermatologie, Pathologie, Serologie, Neurologie aufgeworfen; doch können sie erst zum geringsten Teil beantwortet werden. Außerordentlich wichtig ist das Kapitel über, ,Schwangersehaft und Haut”. Die mannigfachsten Krankheitsbilder werden gezeichnet. Dies und die übrigen, kleineren Kapitel zeigen die große Mannigfaltigkeit der Beziehungen zwischen Haut und Genitale, deren eigentliche Bedeutung jedoch noch clunkel ist, wenn auch für viele Fälle eine veränderte Tätigkeit oder vollständiger Ausfall der Ovarien in Betracht gezogen werden muß. $\quad$ Ernst Puppet. Hug o Schroeder, Das Problem der Unehelichen. (Monographien zur Frauen-kunde und Konstitutionsforschung. IS Гr. 8.) Leezig 1924, Curt Kabitzsch. Weite Kreise verhalten sich noch heute dem illegitimen Kinde und seiner Mutter gegenüber intolerant, weil sie dieses erstere für minderwertig halten und in vollkommener Unkenntnis der sozialen Verhältnisse die un-eheliche Mutter verdammen. Verf. weist an einer großen Anzahl von Sta-tistiken nach, daß das iПegitime Kind dem legitimen weder körperlich noch seelisch nachsteht, daß sein so häufiger Niedergang lediglich exogenen Faktoren zuzuschreiben ist. Vom bevölkerungspolitischen Standpunkt aus betrachtet, müssen wir bei der wachsenden Abnahme der ehelichen Frucht-barkeit, die lediglich axif den Zeugungsunwillen zurückzuführen ist, den Unehelichen eine ganz andere Stellung geben, als sie sie bisher gesellschaft-lich und rechtlich genießen. 252

Literaturverzeichnis.

Das Schicksal der Ledig-Schwangeren ist: ,,Abhängigkeit, Wurzel-losigkeit, Mittellosigkeit. Dieses Geschick verdüstert sich sonderlich in der Zeit, wenn die Entbindung naht, und spricht sich teilweise in der Früh-, Fehl- und Totgeburtenquote aus."

Das Geschick des illegitimen Kindes mit der baldigen Trennung von der Mutter, dem Wechsel der Pflege wird statistisch scharf beleiichtet. Am besten sind die adoptierten und die in der Familie und bei der Mutter aufwachsenden daran. Der Alimentationspflicht genügt nur ein kleiner Teil der Erzeuger; die Vormundschaft sollte eine Berufsvormundschaft werden.

Katastrophal wirken die Wohnungsverhältnisse und der Mangel an ärzt-licher Versorgung, dies namentlich auf dem Lande.

Wenn die Unehelichen in der Krirninalstatistik dominieren, so liegt die Schuld auch nur am Milieu der Erziehung, der mangelhaften Alimentation und der Wohnungsnot.

Verf. halt mit seiner dankenswerten, auf fleißigem Quellenstudium und warmer Anteilnahme für die Ärmsten der Armen beruhenden Arbeit der heutigen Gesellschaft einen Spiegel vor, aus dem sich kein Schatten wegwischen läßt. Hier spricht ein Volkswirtschaftler, den zu hören und zu verstehen auch der heutige Staat alle Veranlassung hat.

Ernst Puppel-Jena.

Hirsch, P.: Die Abderhalden-Reaktion mittels der quantitativen „Inter-ferometrischen Methode” nach P. Hirsch-Jena. Berlin 1925. Julius Springer.

Die Arbeit stellt einen erweiterten Sonderabdruck aus der „Klinischen

Wochenschrift" 1925, Nr. 28 u. 29 dar. A. Martin.

llunge, Ernst, Die Gynäkologie des praktischen Arzíes. 2. Auflage. Berlin und Wien 1925. Urban \& Schwarzenberg.

Kaam ein Jahr ist verstrichen, und die außerordentlich brauchbare Zusammenstellung der für den Praktiker wichtigen gynäkologisehen Fälle erlebt ihre zweite Auflage. Diese Tatsache ist der beste Beweis für den Erfolg des Buches. In der zweiten Auflage ist im Text nur relativ wenig 
geändert worden. Der Leser wird die mannigfaltigen Verbesserungen in den Skizzen, welche die verschiedenen Fälle erläutern, dankbar begrüßen. Sie tragen jedenfalls erheblieh dazii bei, dem praktischen Arzte wie jedem, der sich in cliese nutzbringende Literatur vertiefen will, die einzelnen Krankheiteii näli erz ubr ingen.

V. Literaturverzeichnis.

Geburtshilfe.

Abderhalden, Die Belastungsprobe als diagnostisches Hilfsmittel und Weg zu einer kausalen Therapie. Mecl. Klin. Nr. 48. S. 1792. Abel, Zur Bekämpfung der Wehenschwäche. Dtsch. med. Woch. Nr. 47.

S. 1952. Baumm, H., Zur Frage der rektalen Untersuchung durch die Hebammen.

Munch, mecl. Woch. Nr. 48. S. 2059. Bijlsma, U. G., Die physiologische Wertbestimmung von Hypophysen-

präparaten. (Autoreferat.) Klin. Woch. Nr. 50. S. 2421. 